\title{
Inhaltsverzeichnis
}

Vorwort zur deutschen Ausgabe . . . . . . . . . . . . . . 5

Vorwort ...................... 7

TEIL I

Das Ethnos als dy namisches System

ERSTES KAPITEL

Uber die Stellung des Ethnos im System der menschlichen Gemeinschaften . . . . 13

ZWEITES KAPITEL

Ethnos und ethnosozialer Organismus . . . . . . . . . . . . . . . 35

DRITTES KAPITEL

Die Kultur und ihre ethnischen Funktionen . . . . . . . . . . . . . 45

VIERTES KAPITEL

Zur Frage der ethnischen Aspekte der Psyche . . . . . . . . . . . . 73

FUNFTES KAPITEL

Die Endogamie als „Stabilisator des Ethnos“. Ethnos und Population . . . . . . 106

SECHSTES KAPITEL

Versuch einer Typologisierung ethnischer Gemeinschaften . . . . . . . . . 116

SLEBENTES KAPITEL

Ethnische Prozesse ................... 141

TEIL II

Die Ethmographie als Wissenschaft von den ethnischen Gemeinschaften

ERSTES KAPITEL

Einige Traditionen der Wissenschaft von den Völkern . . . . . . . . . . 165

ZWEITES KAPITEL

Objekt und Gegenstand der ethnographischen Forschung . . . . . . . . . . 189 


\section{DRITTES KAPITEL}

Abgrenzung und Kooperation der Ethnographie zu anderen Wissenschaftsdisziplinen bei der Erforschung der Hauptkomponenten des Ethnos . . . . . . . . . 198

\section{VIERTES KAPITEL}

Die ethnographische Erforschung der Vergangenheit und der Gegenwart. Ethnographie, Geschichte und Soziologie . . . . . . . . . . . . . . . . . 217

FUNFTES KAPITEL

Die Bedeutung der Demographie, Anthropologie und Geographie für die Erforschung ethnischer Gemeinschaften . . . . . . . . . . . . . . . . . 234

Statt eines Schlusses . . . . . . . . . . . . . . . . . . . . . . 242

Bibliographie der verwendeten Literatur in Kyrilliza . . . . . . . . . . . . . 250

Abkürzungen . . . . . . . . . . . . . . . . . . . . . . . 280 